\title{
The Influence of Family Education Mode on Personality Development of Pre-school Children in China
}

\begin{abstract}
Xintong Cai ${ }^{* 1}$
\author{
${ }^{1}$ Beijing Institute of Technology, Zhuhai \\ *Corresponding author. Email: 1035310498@qq.com
}

ABSTRACT

Family education has always been the focus of educator in China. It is the basis of development of individual personality. Parents are the first teachers of children, diverse family education modes will have different impacts on the personality of children. But the majority of literature in this area has been using a single theory and lack of detailed age groups. About these gaps, the author will research the theories of Baumrind (Diana Baumrind,1927) and Erikson (Erik.H.Erikson,1902), and try to discuss the influence of family education modes on personality development of preschool children in China by contrastive analysis. During the analysis, the conclusion is different education modes have different impact on personality of pre-school children. Parents should find a perfect path to help them.
\end{abstract}

Keywords: Family education, Personality development, Pre-school children, Erikson, Diana Baumrind

\section{INTRODUCTION}

In China, people always take education seriously. Preschool education is the foundation stone of all education. But there are still many problems with Chinese family education. With the high speed development of society, the competitiveness of irrational still increases. Parents do not want their child defeated to go up in the scratch line, so they always be strict with their children. For example, the pressure of children are increasing because of afterschool classes and suppressing their nature. The Chinese Ministry of Education issued a document about Guidelines from the Ministry of Education on Strengthening the child discipline in 2015 [1]. This document indicated parents are the first teachers of children and they should pay attention to family education. With this policy, more scholars start researching this area.

In the area of family education that authors want to research, the application of theory in most literature is too simplistic and lacks detailed age groups. The majority of literature is based on the theory of Baumrind. She divides family education mode into four styles: Authoritarian, Permissive, Neglectful and Authoritative[2], every education mode has different characteristics. The author will add a new theory of Erikson to analyze the influence of family education mode on personality development of pre-school children in China. Erikson divided formation and development of personality into eight sections. Different stages have different psychological needs, the influence of family education mode on personality development is also diverse[3]. This paper focuses on the first three phases of this process.

Is there any relationship between family education mode and personality development of pre-school children in China? When parents respond to different psychological needs of children in different ways of education mode, will it have all negative impacts on the development of personality? The author wants to discuss these questions. This paper will research this topic and give a thought and summary. Different theories can help readers to know the significance of family education mode on personality development of pre-school children in China in different perspectives.

\section{THEORY AND CHARACTERISTICS OF PERSONALITY DEVELOPMENT OF PRE- SCHOOL CHILDREN}

Erikson is a famous American psychologist in the 1990s, he divided formation and development of personality into eight sections: Infant, Children, Early school, School age, Adolescent, Such early adulthood, Adult stage and Mature period[3]. Every stage has 
different psychological needs, different social cultures which people can expose to will have the special impact on development of personality stage. In this paper, the author will research the first three phases---Infant, Children and Early school.

\subsection{The Infant (0-1.5)}

In infant, lack of trust is the most conspicuous feature of children.[4] Babies contact their mothers for a long time so they can gain trust and rely on them. When mothers left babies or other people try to approach them, they will feel insecurity and cry. Therefore, building a relationship of trust between infants and family members is the most important target in this stage. If family wants to train the trust of baby, they should create a comfortable environment with love and tolerance, in order to make infants feel trustworthy about the environment and people.

\subsection{The Children (1.5-3)}

As they get older, children begin to learn basic skills, such as crawling and walking. At the same time, they have the ability of independent exploration. However, they did not know how to use the skills correctly, and parents must control and guide them. In this period, the behavior and attitude of parents will impact the contradiction and competitive between autonomy, selfdoubt and shyness in personality of child[4].

\subsection{The Early School (3-5)}

"Competition between initiative and guilt"[4], which is a summary of the aim in this stage by Erikson. Compared with the past two phases, curiosity of children becomes higher. They want to explore the world with the help of parents or their own. In the meantime, the curiosity also promotes the emergence and development of imagination and creativity of children. That is why children like to ask their parents about some childish and interesting questions. In this period, the way of parents respond to the questions or behaviors of children also determines the direction of personality development.

From the above-mentioned several aspects, it is observed that the development of personality of children is related to the way of family education mode. Any kinds of education modes will have different influence on the formation and development of personality of pre-school children. The following sections will describe the topic on the basis of the theories of Baumrind and Erikson.

\section{THE FEATURE OF DIFFERENT FAMILY EDUCATION MODES AND THE INFLUENCE ON PERSONALITY DEVELOPMENT OF PRE-SCHOOL CHILDREN}

\subsection{Authoritarian}

Authoritarian parents are obsessed with control and refuse to communicate equality with children. In this category, parents give their children few warmth and tolerance, they force children to obey them and let them to do something that parents think it is correct. In addition, parents intervene too much in their children's thoughts and behaviors and do not respect their children's needs. Suppressing the nature and novel ideas by children makes them lose their private space and the ability to think about themselves[2].

In infant, the body and language ability of children has underdeveloped, they only use crying to attract their parents to meet the physical and emotional needs. At this stage, autocratic parents can absolutely control the lives of children, which can satisfy their need of control. As for the emotional needs, parents of family education mode can not have enough tolerance and response, they tend to be impatient with sobbing child. This will make children feel less emotional feedback from their parents. In this basic of crisis of confidence, children are easy to produce some negative emotions, such as social phobia and anxiety.

After children enter into the stage of children and early school, their independent consciousness and curiosity and creativity begin to emerge. The authoritarian family start trying to control their thoughts and behaviors, but this control is not reasonable. They want to force children to obey them. So when children can not use their skills correctly and want to express their feelings, what they get is not positive feedback, but dissatisfaction and depression from their parents. Children who receive feedback for negative for a long time will be suspicious and ashamed of their own thoughts and behaviors, which can make them afraid to express themselves. The children who develop their personality in this family education mode mostly have negative personality, such as suspicion, shyness and guilt. They are cowardly, and lack of willpower and confidence. Some of children become traitorous and revolt against parental disciplines. All the research results show that the authoritative family education model is easy to affect the personality development of preschool children in China.

\subsection{Permissive}

The characteristic of Permissive parents are responsive. Children can obtain a lot of warmth and 
encouragement from parents, and all the emotions are tolerated. However, parents do not have reasonable control strategies and methods with the behavior and requirements of children. They only satisfy their children as much as possible in material,but not in psychological training and guidance. Children only do something according to their feelings and cognition when they are unable to make decisions without the help of parents[2].

In the stage of infant, permissive parents can give children enough tolerance and understanding when they lack of affection. They communicate with their child by friendliness speaking and behaviors, and response their needs of materially and psychological positively. When children crying because of their physical or emotional needs, permissive parents can help them to solve their problem with love and patience. In this environment filled with warmth, personality of pre-school children do not lack of trust. They can adapt to unfamiliar environment quickly and begin to connect with others because parents give them enough emotional feedback.

In the next two phases, the love from permissive parents will promote the development of autonomy and creativity of child personality. When children cannot judge whether their behavior is correct or not, and cannot make decisions by themselves, the control and guidance of parents is the most important link. But permissive parents will not control it in rational, they indulge their child in growth. If children accept the uncontrolled love from their parents for a long time, they will form a personality that is self-centered, and do not consider and respect others because of their excessive autonomy. In this family education mode, the personality of pre-school children will be repelled by other people because of their egoism and disrespect.

\subsection{Neglectful}

The characteristic of Neglectful education mode is indifferent. It has a common trait with permissive education mode: compared with rational control and development for a long time, they prefer to satisfy the material needs of children and remain to ignore the personality development of children. But neglectful parents pay less attention to the emotions of pre-school children, and they ignore the spiritual needs for family affection of child[2]. Children has too much freedom and all the emotions can not getting any response from their parents, which will makes children lose their ability to express themselves.

In infant, children need lots of love in this stage, but apathy is the feature of neglectful education mode. Parents ignore the real emotional needs of children, and they only feel impatient with sobbing child. These negative attitudes will make children not get enough trust and affection because they did not get enough emotional feedback. Babies will lack trust easily in this environment. As same as the children of Authoritarian family education mode, they will develop their personality in a negative direction in the future.

During the period of the children and the early school, parents should spend their time and pay more attention to encouraging children develop independently, and prevent them to making wrong decisions. But Neglectful parents cannot waste their time to communicate with their child. They do not encourage or criticize it. Left-behind children is the tropical case. Parents who work out for a long time cannot satisfy the needs of psychological besides material for children. The pre-schoool children in Neglectful education mode cannot be concerned by their parents, and the emotional export cannot receive the feedback for a long time. It is easy to make children feel guilty and reduce the display of self-emotion. These personalities of children are always introverted, because they have an overwhelming loneliness and unsociable.

\subsection{Authoritative}

Equality is the feature of Authoritative education mode. Authoritative parents give children enough trust, concern and respect their behavior. They communicate with children, reasonably control their own needs, encourage children to make independent decisions, and develop their own nature and independence. At the same time, parents who establish authority, do not lie to their children, and regulate their children's behavior are treated with respect and trust by their children. It can make a positive impact[2].

In the first phase, Authoritative parents can creat a comfortable environment with love and tolerance to train the trust of children. They can spend enough time and take a positive attitude to respond the needs and insecurity of children quickly. For example, keep the kind expressions and words to communicate with baby, encourage them when children begin to self-expression, and comfort their mood and solve their problems. In this environment, personality of pre-school children can get enough emotional feedback, and they will be easily adaptable in the strange environment and establish a good social relationship quickly with strangers.

In the period of children and the early school, this education mode also can guide children develop their positive personality. Authoritative parents can spend enough time leading children to make decisions or solve their problems by themselves, which can develop the sense of autonomy and independence of child and encourage children to develop their nature and correct their errors without delay. When children have any questions with opinion of parents, parents can respect their decision and have an equality exchange. Parents not only give children enough needs, but also carry out the reasonable control. In this equal and democratic family education mode, personality of pre-school children have 
a great prospect of development with autonomy and initiative. It also have some features of extroversion and creativity.

\section{CONCLUSION}

By way of contrastive analysis of the influence of different family education modes on personality development of pre-school children in China, it can prove the accuracy of inference and answer the questions at the beginning proposed by the author.

First of all, family education mode is related to the personality development of preschool children in China. It can interconnect with the theory of Personality Development by Erikson. Analysis in different ways and theories can help people understand the characteristics of personality of pre-school children at each phase. It can prove the importance between family education mode and the development of pre-school children in China. The early childhood education is basic on progress of individual personality, and different education modes have strong impact on it[5].

Second, not all family education modes will have a negative impact on the development of personality of children. The family education mode of Authoritative is positive with equality, tolerance and reasonable. But the other education modes will cause pre-school children lack of trust, autonomy and initiative in personality development in China.

In the research of future, the author will focus on how to popularize the education mode and methodology by Authoritative. It is also important to promote parents build a good framework of personality for their child and improve the personality development of Chinese preschool children.

\section{REFERENCES}

[1] Li Jinjin,Chen Liang. The Influence and Response of Different Family Education Mode on the Formation of Children's Personality[J]. Live Education,2020(07):53-56.

[2] Li Xue.(2019). On the Influence of Family Education on the Formation of Children's Personality. Education Science \& Culture Magazine(07),127128+170. doi:10.16871/j.cnki.kjwha.2019.07.058.

[3] Guo Ting. Discussion of Erikson's theory of stages of personality development[J]. Theory Guides, 2010(06):26-27.

[4] Wang Jiajun. Eriksson' s Theory of Personality Development And the Cultivation of Children' s Healthy Personality[J]. Studies In Preschool Education,2011(06):37-40.
[5] Hao Hongying. Enlightenment of Erickson' s Personality Development Theory to Family Personal Education[J]. Journal of Yinshan, 2008(04):122-125. 